\title{
Delayed Catabolism of High Density Lipoprotein Apolipoproteins A-I and A-II in Human Cholesteryl Ester Transfer Protein Deficiency
}

\author{
Katsunori Ikewaki, " Daniel J. Rader, " Takuya Sakamoto," Masato Nishiwaki," Naoki Wakimoto," Juergen R. Schaefer," \\ Toshitsugu Ishikawa, Thomas Fairwell, ${ }^{*}$ Loren A. Zech, * Haruo Nakamura, ${ }^{*}$ Makoto Nagano, ${ }^{*}$ and H. Bryan Brewer, Jr." \\ *Molecular Disease Branch, National Heart, Lung and Blood Institute, National Institutes of Health, Bethesda, Maryland 20892; \\ ‡Department of Internal Medicine, Aoto Hospital, Jikei University School of Medicine, Tokyo, Japan, \\ ${ }^{\S}$ First department of Internal Medicine, National Defense Medical College, Saitama, Japan; \\ and "Department of Internal Medicine, Sapporo General Hospital, Hokkaido, Japan
}

\begin{abstract}
Deficiency of the cholesteryl ester transfer protein (CETP) in humans is characterized by markedly elevated plasma concentrations of HDL cholesterol and apoA-I. To assess the metabolism of HDL apolipoproteins in CETP deficiency, in vivo apolipoprotein kinetic studies were performed using endogenous and exogenous labeling techniques in two unrelated homozygotes with CETP deficiency, one heterozygote, and four control subjects. All study subjects were administered ${ }^{13} \mathrm{C}_{6}$-labeled phenylalanine by primed constant infusion for up to $16 \mathrm{~h}$. The fractional synthetic rates (FSRs) of apoA-I in two homozygotes with CETP deficiency $(0.135,0.134 / d)$ were found to be significantly lower than those in controls $(0.196 \pm 0.041 / d, P<0.01)$. Delayed apoA-I catabolism was confirmed by an exogenous radiotracer study in one CETP-deficient homozygote, in whom the fractional catabolic rate of ${ }^{125}$ I-apoA-I was 0.139 / d (normal $0.216 \pm 0.018 / d)$. The FSRs of apoA-II were also significantly lower in the homozygous CETP-deficient subjects $(0.104,0.112 / d)$ than in the controls $(0.170 \pm 0.023 / d, P$ $<0.01)$. The production rates of apoA-I and apoA-II were normal in both homozygous CETP-deficient subjects. The turnover of apoA-I and apoA-II was substantially slower in both $\mathrm{HDL}_{2}$ and $\mathrm{HDL}_{3}$ in the CETP-deficient homozygotes than in controls. The kinetics of apoA-I and apoA-II in the CETP-deficient heterozygote were not different from those in controls. These data establish that homozygous CETP deficiency causes markedly delayed catabolism of apoA-I and apoA-II without affecting the production rates of these apolipoproteins. ( $J$. Clin. Invest. 1993. 92:1650-1658.) Key words: cholesteryl ester transfer protein • kinetics • stable isotopes • high density lipoproteins $\bullet$ atherosclerosis
\end{abstract}

\section{Introduction}

Epidemiologic studies have consistently demonstrated that plasma concentrations of HDL cholesterol (HDL-C) ${ }^{1}$ are in-

A portion of this paper was presented at the American Heart Association meeting held in Anaheim, CA, November 1991.

Address correspondence to Katsunori Ikewaki, M.D., Building 10, Room 7N1 17, National Institutes of Health, 9000 Rockville Pike, Bethesda, MD 20892.

Received for publication 13 January 1993 and in revised form 15 April 1993

1. Abbreviations used in this paper: CE, cholesteryl ester; CETP, cholesteryl ester transfer protein; FCR, fractional catabolic rate; FSR, fractional synthetic rate; HDL-C, HDL cholesterol; PR, production rate; $\mathrm{RT}$, residence time.

The Journal of Clinical Investigation, Inc.

Volume 92, October 1993, 1650-1658 versely correlated with the incidence of coronary heart disease (CHD) $(1,2)$. Although the mechanisms by which HDL protects against atherosclerosis remain uncertain, HDL has been postulated to facilitate the efflux of cholesterol from peripheral tissues and transport it back to the liver in a process termed reverse cholesterol transport (3-5). The major protein constituents of HDL are apoA-I and apoA-II (6). Plasma concentrations of apoA-I are inversely correlated with CHD risk, whereas the association of apoA-II levels with CHD is not well established (7). Kinetic studies have established that plasma apoA-I levels are correlated with apoA-I catabolic rates, whereas plasma apoA-II levels are linked to apoA-II production rates in normolipidemic subjects $(8-10)$. However, the factors regulating the metabolism of these apolipoproteins are not well understood.

Cholesteryl ester transfer protein (CETP) is a major factor modulating plasma HDL-C metabolism (11). CETP, a hydrophobic glycoprotein with a molecular weight of $74 \mathrm{kD}$, catalyzes the transfer of lipids among lipoproteins $(11,12)$. In humans, CETP-mediated transfer of cholesteryl ester (CE) from HDL to apoB-containing lipoproteins may be a major route of reverse cholesterol transport (13). The recent discovery that humans with CETP deficiency have markedly increased HDL cholesterol and apoA-I levels (14-17) supports the concept that CETP is an important factor regulating plasma HDL-C concentrations. However, the mechanism by which deficiency of CETP results in elevated apoA-I levels has not been established. To gain insight into the role of CETP in HDL apolipoprotein metabolism, we performed in vivo kinetic studies of apoA-I and apoA-II in two unrelated homozygotes with CETP deficiency, one heterozygote, and control subjects using both endogenous stable isotope and exogenous radiotracer techniques. The data establish that the catabolism of both apoA-I and apoA-II is significantly delayed in CETP deficiency.

\section{Methods}

Study subjects. Two unrelated homozygotes with CETP deficiency, one heterozygote, and four control subjects were investigated. The CETP-deficient subjects were referred to the National Defense Medical College, Saitama (subject 1) or Jikei University School of Medicine, Tokyo (subject 2) with markedly elevated plasma HDL-C levels. In both homozygous patients, plasma apoA-I levels were also increased, whereas apoA-II levels were in the upper range of normal. No CETP activity was detected in their plasma by a modification of the method by Tollefson and Albers ( 18). Sequencing of appropriate regions of the CETP gene after amplification by the polymerase chain reaction (19) established that both subjects were homozygous for the $G$ to A substitution at the 5 -splice donor site of intron 14, which has been reported previously $(17,20,21)$. A son of CETP-deficient subject 2 , an obligate heterozygote, had an HDL-C of $61 \mathrm{mg} / \mathrm{dl}$. The $\mathrm{HDL}_{2}-\mathrm{C}$ levels in the CETP-deficient subjects were selectively increased, whereas $\mathrm{HDL}_{3}-\mathrm{C}$ 
levels were not different from controls. Consistent with previous reports $(20,22)$, the $\mathrm{HDL}_{2}-\mathrm{C} / \mathrm{HDL}_{3}-\mathrm{C}$ ratios of the CETP-deficient homozygotes and the heterozygote were increased to $9.2,12.3$, and 3.0, respectively (normal $0.4 \pm 0.2$ ). The molecular weights and isoelectric points of apoA-I and apoA-II were normal by SDS-PAGE and isoelectric focusing (23).

The clinical characteristics and lipid values of the study subjects are shown in Table I. Values are the mean of five fasting determinations made during the study. Plasma lipid and apolipoprotein concentrations remained in steady state throughout the study period. The four control subjects were healthy young females (mean age of $21 \mathrm{yr}$ ) and had normal fasting lipid and apolipoprotein levels. All study subjects had normal fasting plasma glucose levels and normal thyroid, hepatic, and renal function. The study protocol was approved by the Internal Review Boards of Jikei University School of Medicine and the National Heart, Lung and Blood Institute. Informed consent was given by each of the participants.

Endogenous stable isotope study protocol. After a 12-h fast, ${ }^{13} \mathrm{C}_{6}$-phenylalanine $\left(99 \%{ }^{13} \mathrm{C}_{6}\right.$, Cambridge Isotope Laboratories, Woburn, MA) was administered as a priming bolus of $600 \mu \mathrm{g} / \mathrm{kg}$, immediately followed by a constant infusion of $12 \mu \mathrm{g} / \mathrm{kg}$ per min for up to 12 to $16 \mathrm{~h}$. Blood samples $(20 \mathrm{ml})$ were obtained from the opposite arm at $10 \mathrm{~min}, 1,2,3,4,5$, and $6 \mathrm{~h}$, and every $2 \mathrm{~h}$ thereafter until the end of infusion. During the infusion meals were served in equal small portions every $2 \mathrm{~h}$. Plasma was separated by centrifugation at $2,300 \mathrm{rpm}$ for 30 $\min$ at $4^{\circ} \mathrm{C}$.

Exogenous radiotracer study. ApoA-I was isolated from normal HDL ( 24) and stored at $-20^{\circ} \mathrm{C}$. Lyophilized apolipoprotein was solubilized in a buffer of $6 \mathrm{M}$ guanidine- $\mathrm{HCl}$ and $1 \mathrm{M}$ glycine ( $\mathrm{pH} \mathrm{8.5)} \mathrm{and}$ iodinated with ${ }^{125} \mathrm{I}$ by a modification of the iodine monochloride method, as previously reported (25). Iodination efficiency was $40 \%$ with incorporation of $\sim 0.5$ mole iodine per mole of protein. ${ }^{125} \mathrm{I}$ apoA-I was reassociated with autologous lipoproteins and dialyzed extensively to remove free iodine. Human serum albumin was added to a final concentration of $5 \%(\mathrm{wt} / \mathrm{vol})$. The injection samples were sterilefiltered through a $0.22-\mu \mathrm{m}$ filter (Millipore Continental Water Systems, Bedford, MA) and tested for pyrogens and sterility. One day before the study, the homozygous CETP-deficient subject 1 was given potassium iodide $(900 \mathrm{mg})$ in divided doses and this was continued throughout the study period. After a 12 -h fast, $50 \mu \mathrm{Ci}$ of ${ }^{125} \mathrm{I}$-apoA-I was injected into the CETP-deficient subject simultaneously with the stable isotope protocol above. Blood samples were obtained $10 \mathrm{~min}$ after the injection and then at selected time points through $14 \mathrm{~d}$. Urine was collected continuously throughout the study. Sodium azide and apro- tinin were added to the plasma at a final concentration of $0.05 \%$ and $200 \mathrm{KIU} / \mathrm{ml}$, respectively. Radioactivity in plasma and urine was quantitated in a gamma counter (Cobra; Packard Instrument Co., Downers Grove, IL).

Isolation of lipoproteins. Total plasma lipoproteins were isolated from the plasma sample obtained at different time points after injection by ultracentrifugation at a density of $1.25 \mathrm{~g} / \mathrm{ml}$ in a tabletop ultracentrifuge (TL-100; Beckman Instruments, Palo Alto, CA ) using TLA100.3 rotor at $100,000 \mathrm{rpm}$ at $10^{\circ} \mathrm{C}$ for $10 \mathrm{~h}$. Of total plasma $97.3 \%$ and 97.4\% apoA-I and apoA-II were present in the top fraction. VLDL, IDL, LDL, $\mathrm{HDL}_{2}$, and $\mathrm{HDL}_{3}$ were isolated by sequential ultracentrifugation from $5 \mathrm{ml}$ of plasma as previously described (26). In the homozygous CETP-deficient subjects, lipoproteins in the $d=1.019-1.063$ $\mathrm{g} / \mathrm{ml}$ range consisted of $\mathrm{LDL}$ as well as apoA-I-containing lipoproteins. These apoA-I-containing particles do not have apoB but are rich in apoE and have been referred to as $\operatorname{HDL}_{1}(27,28)$. To isolate the $\mathrm{HDL}_{1}$ fraction, a polyclonal antiserum against apoB (BoehringerMannheim, Mannheim, Germany) was coupled to CNBr-Sepharose $(5 \mathrm{mg} / \mathrm{g} \mathrm{gel}$ ) as reported previously (25) and lipoproteins of $d=1.019$ $1.063 \mathrm{~g} / \mathrm{ml}$ from the homozygous CETP-deficient subjects were applied to this anti-apoB immunoaffinity column. The nonretained fraction $\left(\mathrm{HDL}_{1}\right)$ was collected and apolipoprotein composition was analyzed by SDS-PAGE.

Isolation of apolipoproteins. Lipoproteins were dialyzed against 10 $\mathrm{mM}$ ammonium bicarbonate, lyophilized, and delipidated. VLDL apoB- 100 and total, $\mathrm{HDL}_{1}, \mathrm{HDL}_{2}$, and $\mathrm{HDL}_{3}$ apoA-I were isolated by preparative gradient SDS-PAGE (5-15\%) as previously reported (29). Total, $\mathrm{HDL}_{2}$, and $\mathrm{HDL}_{3}$ apoA-II were isolated by preparative isoelectric focusing ( $\mathrm{pH} 4-6.5)$ (30). Apolipoproteins were identified by coelectrophoresis of purified apoA-I and apoA-II standards as well as by immunoblotting using monoclonal antibodies against apoB-100, apoA-I, and apoA-II.

Determination of isotopic enrichment. Samples were prepared for gas chromatography-mass spectrometry analysis as reported previously (29). Briefly, apolipoprotein bands were cut from gels, dried overnight $\left(90^{\circ} \mathrm{C}\right)$, and subjected to hydrolysis in $6 \mathrm{~N} \mathrm{HCl}$ (Ultrapure grade; J. T. Baker, Inc., Phillipsburg, $\mathrm{NJ}$ ) at $110^{\circ} \mathrm{C}$ for $24 \mathrm{~h}$. The protein hydrolysates were lyophilized in an evaporator (Speed-Vac; Savant Instrument, Inc., Farmingdale, NY). Plasma free amino acids and protein hydrolysates were purified by cation exchange chromatography (AG-50W-X8; Bio-Rad Laboratories, Richmond, CA). Recovered amino acids were derivatized to the $N$-heptafluorobutyryl isobutyl esters, dissolved in ethyl acetate, and analyzed by gas chromatographymass spectrometry (4500; Finnigan MAT, San Jose, CA ) in the chemi-

Table I. Characterization of Study Subjects

\begin{tabular}{|c|c|c|c|c|c|c|c|c|}
\hline Subjects & Sex & Age & BMI & TC & TG & HDL-C & ApoA-I & ApoA-II \\
\hline & & $y r$ & $\mathrm{~kg} / \mathrm{m}^{2}$ & & & $m g / d l$ & & \\
\hline \multicolumn{9}{|l|}{ CETP deficiency } \\
\hline \multicolumn{9}{|l|}{ Homozygotes } \\
\hline 1 & $\mathbf{M}$ & 42 & 26.0 & 251 & 98 & 177 & 210 & 37 \\
\hline 2 & $\mathrm{~F}$ & 67 & 18.7 & 282 & 79 & 170 & 216 & 39 \\
\hline Heterozygote* & $\mathbf{M}$ & 40 & 24.2 & 145 & 67 & 61 & 126 & 29 \\
\hline \multicolumn{9}{|l|}{ Controls } \\
\hline 1 & $\mathrm{~F}$ & 21 & 19.3 & 176 & 61 & 53 & 128 & 27 \\
\hline 2 & $\mathrm{~F}$ & 22 & 22.0 & 185 & 96 & 53 & 138 & 37 \\
\hline 3 & $\mathrm{~F}$ & 21 & 21.9 & 144 & 62 & 49 & 131 & 28 \\
\hline 4 & $\mathrm{~F}$ & 20 & 19.3 & 163 & 40 & 66 & 140 & 31 \\
\hline Mean & & 21 & 20.6 & 167 & 65 & 55 & 134 & 31 \\
\hline SD & & 0.7 & 1.3 & 15 & 20 & 6 & 5 & 4 \\
\hline
\end{tabular}

* The CETP-deficient heterozygote is the son of the homozygous CETP-deficient subject 2. BMI; body mass index; TC, total cholestero; TG, triglycerides. 
cal ionization mode, using isobutane as the reagent gas. Selective ion monitoring at $418 \mathrm{~m} / \mathrm{z}$ for unlabeled phenylalanine and $424 \mathrm{~m} / \mathrm{z}$ for ${ }^{13} \mathrm{C}_{6}$-phenylalanine was used to determine the isotope ratio. Each sample was analyzed at least three times. Enrichment was calculated from isotope ratio using the method by Cobelli et al. (31). The enrichment was then converted to the tracer/tracee ratio by using the formula: tracer/tracee ratio $=e(t) /\left[e_{\mathrm{i}}-e(t)\right]$, where $e(t)$ is the enrichment of each sample at time $t$, and $e_{\mathrm{i}}$ is the enrichment of the infusate (31). In this study, $e_{\mathrm{i}}$ was 0.99 .

Determination of kinetic parameters. For the endogenous labeling studies, the tracer/tracee ratio data of VLDL apoB-100, apoA-I, and apoA-II were fitted to monoexponential functions using SAAM 30 (32). The length of the constant infusion did not permit the use of the more than one exponential in the kinetic analysis of the data. The function was defined as: $A(t)=A_{\mathrm{p}}\left(1-e^{-(k(t-d))}\right)$, where $A(t)$ is the tracer/tracee ratio at time $t, A_{\mathrm{p}}$ is the precursor pool tracer/tracee ratio for the apolipoprotein, $k$ is the fractional synthetic rate (FSR), and $d$ is the delay. The tracer/tracee ratios of VLDL apoB-100 and total apoA-I and apoA-II were simultaneously analyzed using the VLDL apoB-100 plateau tracer/tracee ratio as the estimate of the precursor pool enrichment for VLDL apoB-100 and plasma apoA-I and apoA-II. We have found that apoA-I FSRs determined by monoexponential analysis of endogenous labeling data using the VLDL apoB-100 plateau tracer/ tracee ratio as an estimate of the precursor pool tracer/tracee ratio for apoA-I synthesis were in excellent agreement (4\% difference, $P=\mathrm{NS}$ ) with those obtained simultaneously by the exogenous radiotracer method (Ikewaki, unpublished data). Apolipoprotein production rates (PR) were determined using the formula: $P R=(F S R) \times($ plasma apolipoprotein concentration $) \times($ plasma volume $) /($ body weight $)(33$, 34). Plasma volume was assumed to be $4 \%$ of body weight.

For the exogenous radiotracer studies, plasma radioactivity curves were constructed by dividing the plasma radioactivity at each time point by the plasma radioactivity at the initial 10 -min time point. The curves were fitted to biexponential functions using SAAM 30 (32). The length of the radiotracer study allowed the use of the biexponential in the kinetic analysis of the data. Residence times were obtained from the area under the curve. The fractional catabolic rate (FCR) is the reciprocal of the residence time. At steady state, the FCR is equal to the FSR. Despite the differences in data analysis between the two methods, the apoA-I kinetic parameters in one of the homozygous CETP-deficient patients were virtually identical.

Analytical methods. Total cholesterol and triglyceride levels were determined by automated enzymic techniques on an analyzer (VPSS; Abbott Laboratories, North Chicago, IL). HDL cholesterol was measured by dextran sulfate precipitation (35). Plasma apoA-I and apoAII concentrations were quantitated using turbidimetric assays (Boehringer-Mannheim, Mannheim, Germany). ApoA-I concentrations in $\mathrm{HDL}_{1}$ and LDL separated by anti-apoB immunoaffinity column were quantitated by ELISA as previously reported (36). Laser scanning densitometry (Ultrascan XI; LKB, Piscataway, NJ) of SDS-PAGE gels was used to determine the relative content of apoA-I and apoE in $\mathrm{HDL}_{1}$.

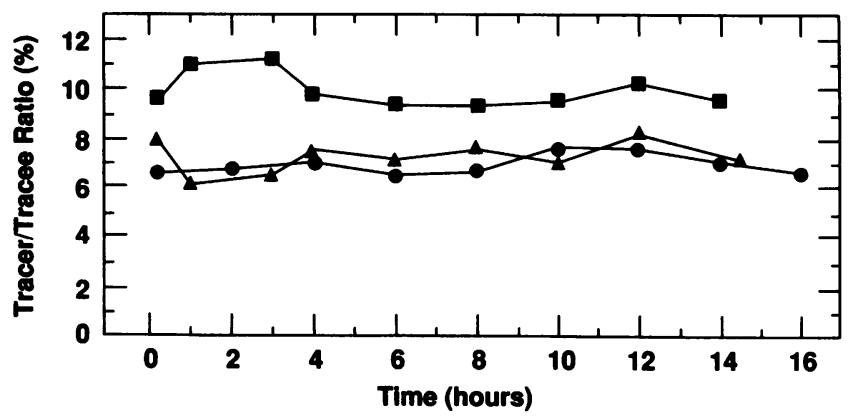

Figure 1. Tracer/tracee ratios of free plasma phenylalanine in homozygous CETP-deficient subject 1 (circles) and subject 2 (squares) and a heterozygous CETP-deficient subject (triangles).

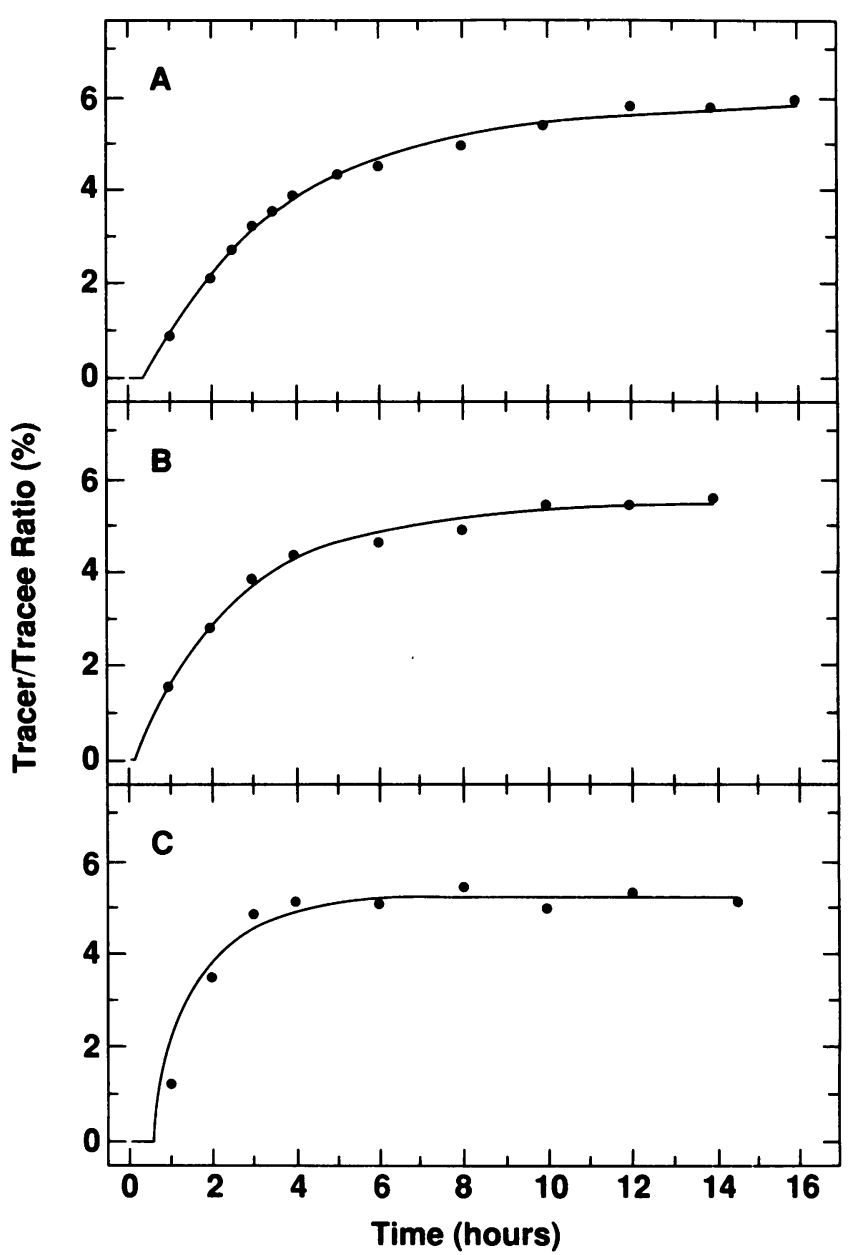

Figure 2. Tracer/tracee ratios of VLDL apoB-100 in homozygous CETP-deficient subject $1(A)$ and subject $2(B)$ and a heterozygous CETP-deficient subject $(C)$. Data were fitted by monoexponential function analysis as described in the methods section.

\section{Results}

The tracer/tracee ratio curves of free plasma phenylalanine during the constant infusion in the two CETP-deficient homozygotes and the heterozygote are illustrated in Fig. 1. In all study subjects, the tracer/tracee ratio of free plasma phenylalanine increased rapidly after the priming bolus, then remained relatively constant throughout the infusion period. The mean tracer/tracee ratios of free plasma phenylalanine were 6.8 and $9.8 \%$ in the homozygous CETP-deficient subjects 1 and 2, respectively, and $7.2 \%$ in the heterozygous subject. The mean tracer/tracee ratio of free plasma phenylalanine in the four control subjects was $5.6 \pm 0.2 \%$.

The tracer/tracee ratios of VLDL apoB-100 during the constant infusion in the CETP deficient subjects are shown in Fig. 2. Monoexponential function analysis determined the VLDL apoB-100 plateau tracer/tracee ratios to be $5.9 \%$ in the homozygous CETP-deficient subject $1,5.4 \%$ in subject 2 , and $5.2 \%$ in the heterozygous subject. The mean VLDL apoB-100 plateau tracer/tracee ratio in the four controls was $5.2 \pm 0.5 \%$.

The apoA-I tracer/tracee ratio curves are illustrated in Fig. 3. In both homozygous CETP-deficient subjects, the apoA-I tracer/tracee ratio curves had shallower slopes than did the 


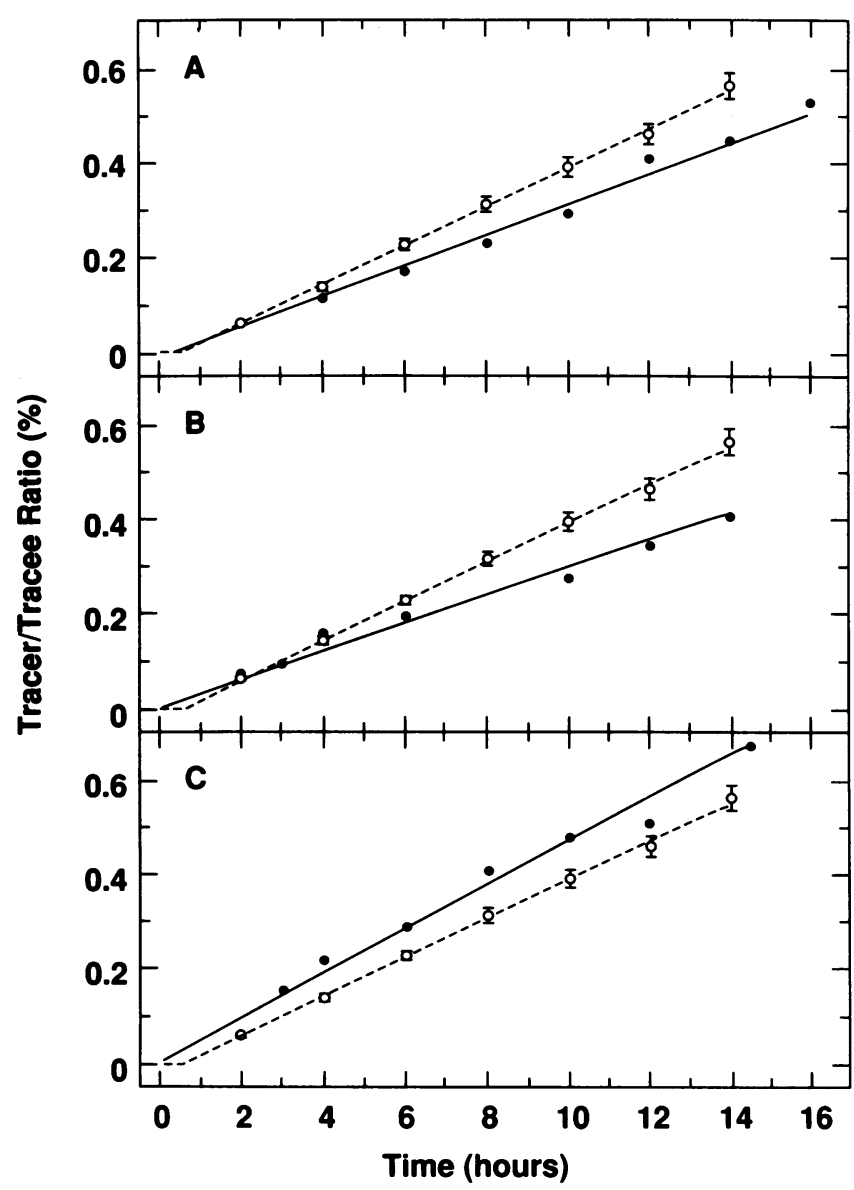

Figure 3. Tracer/tracee ratios of total plasma apoA-I (solid circles) in homozygous CETP-deficient subject $1(A)$ and subject $2(B)$ and a heterozygous CETP-deficient subject $(C)$ with the mean tracer/tracee ratio of control subjects (open circles with dotted line). Data from control subjects are given as the mean \pm SD. Data were fitted by monoexponential function analysis with the VLDL apoB-100 plateau tracer/tracee ratio as the estimate of the precursor pool tracer/tracee ratio for apoA-I.

curve of the mean of four control subjects, indicating a delayed apoA-I catabolism. However, the apoA-I tracer/tracee ratio curve in the heterozygous subject was similar to the mean of

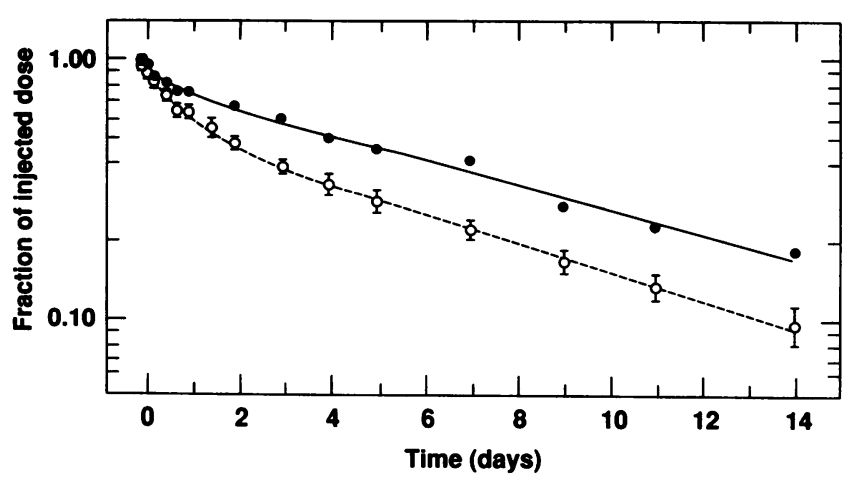

Figure 4. Metabolism of ${ }^{125} \mathrm{I}$-apoA-I in homozygous CETP-deficient subject 1 (solid circles) and the mean of 10 control subjects (open circles with dotted line). Data from control subjects are given as the mean \pm SD. control subjects. The ${ }^{125}$ I-apoA-I plasma curve in homozygous CETP-deficient subject 1 is shown in Fig. 4 with the mean ${ }^{125}$ I-apoA-I plasma curve of 10 normal control subjects. The exogenous apoA-I radiotracer study confirmed the delayed apoA-I catabolism in this CETP-deficient subject. The apoA-II tracer/tracee ratio curves are illustrated in Fig. 5. They also demonstrate shallower slopes in the CETP-deficient homozygotes, consistent with slower turnover of apoA-II in these subjects.

The kinetic parameters of total plasma apoA-I and apoA-II are summarized in Table II. In the two CETP-deficient homozygotes, the mean FSR of total plasma apoA-I was decreased by $31 \%$ compared with that in control subjects $(P<0.01)$. The apoA-I FCR obtained by the exogenous radiotracer method $(0.139 / \mathrm{d})$ was highly comparable with that obtained by endogenous labeling $(0.135 / \mathrm{d})$ in the homozygous CETP-deficient subject 1 . The mean FSR of apoA-II was decreased by $36 \%$ in the CETP-deficient homozygotes compared with that in the controls $(P<0.01)$. As in the control subjects, the apoA-II FSRs were lower than the apoA-I FSRs in both CETP-deficient

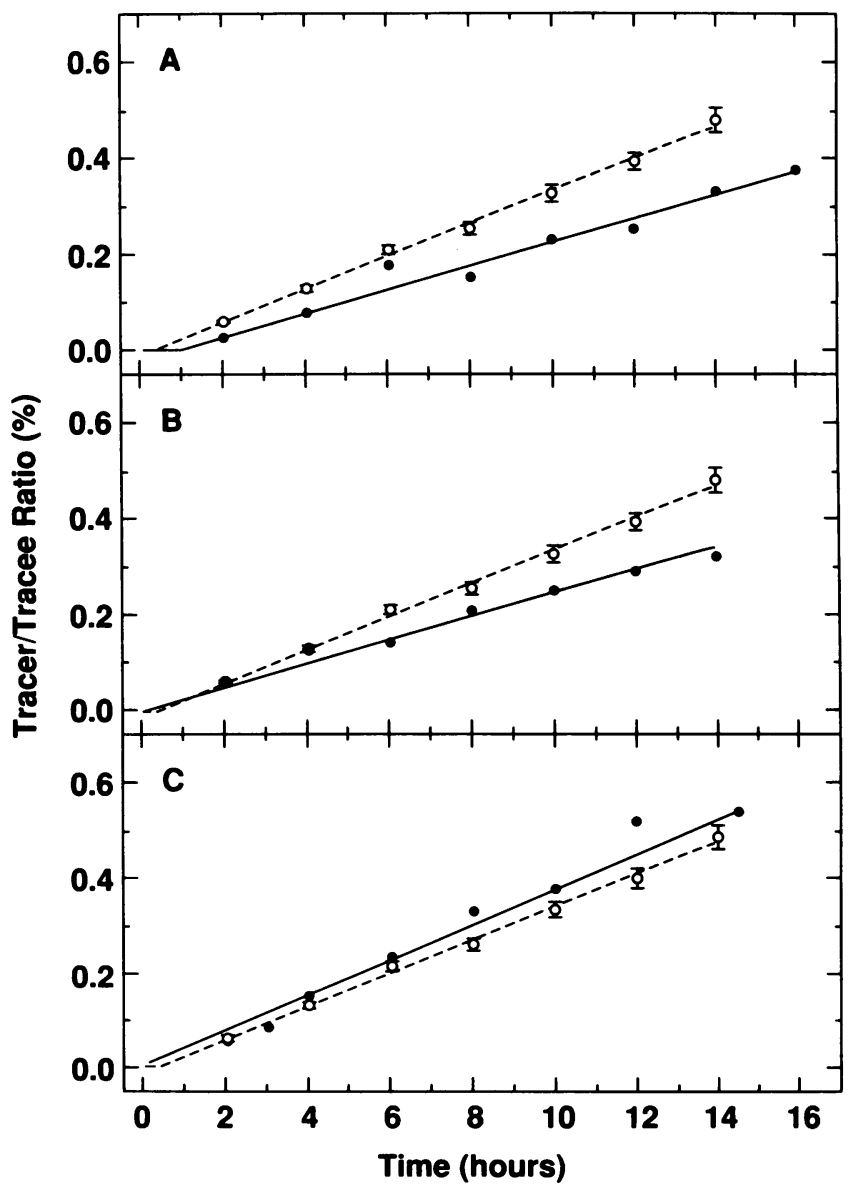

Figure 5. Tracer/tracee ratios of total plasma apoA-II (solid circles) in homozygous CETP-deficient subject $1(A)$ and subject $2(B)$ and a heterozygous CETP-deficient subject $(C)$ with the mean tracer/tracee ratio of control subjects (open circles with dotted line). Data from control subjects are given as the mean $\pm S D$. Data were fitted by monoexponential function analysis with the VLDL apoB-100 plateau tracer/tracee ratio as the estimate of the precursor pool tracer/tracee ratio for apoA-II. 
Table II. Kinetic Parameters of Total Plasma apoA-I and apoA-II in Subjects with CETP Deficiency

\begin{tabular}{|c|c|c|c|c|c|c|}
\hline \multirow[b]{2}{*}{ Subjects } & \multicolumn{3}{|c|}{ ApoA-I } & \multicolumn{3}{|c|}{ ApoA-II } \\
\hline & Conc. & FSR & PR & Conc. & FSR & PR \\
\hline & $m g / d l$ & $d^{-1}$ & $m g / k g-d$ & $m g / d l$ & $d^{-1}$ & $\mathrm{mg} / \mathrm{kg}-\mathrm{d}$ \\
\hline \multirow{2}{*}{\multicolumn{7}{|c|}{$\begin{array}{c}\text { CETP deficiency } \\
\text { Homozygotes }\end{array}$}} \\
\hline & & & & & & \\
\hline 1 & 210 & $\begin{array}{c}0.135 \pm 0.022 \\
(0.139 \pm 0.002)^{*}\end{array}$ & $11.4 \pm 1.8$ & 37 & $0.104 \pm 0.017$ & $1.55 \pm 0.25$ \\
\hline 2 & 216 & $0.134 \pm 0.020$ & $11.6 \pm 1.7$ & 39 & $0.112 \pm 0.017$ & $1.74 \pm 0.26$ \\
\hline Heterozygote & 126 & $0.229 \pm 0.016$ & $11.5 \pm 0.8$ & 29 & $0.182 \pm 0.022$ & $2.11 \pm 0.26$ \\
\hline \multicolumn{7}{|l|}{ Controls } \\
\hline 1 & 138 & $0.217 \pm 0.031$ & $12.0 \pm 1.7$ & 36 & $0.210 \pm 0.030$ & $3.05 \pm 0.43$ \\
\hline 2 & 128 & $0.191 \pm 0.025$ & $9.8 \pm 1.3$ & 27 & $0.153 \pm 0.016$ & $1.64 \pm 0.17$ \\
\hline 3 & 140 & $0.174 \pm 0.062$ & $9.7 \pm 3.5$ & 31 & $0.160 \pm 0.053$ & $1.99 \pm 0.66$ \\
\hline 4 & 131 & $0.201 \pm 0.046$ & $10.5 \pm 2.4$ & 28 & $0.156 \pm 0.027$ & $1.72 \pm 0.30$ \\
\hline Mean \pm SD & $134 \pm 5$ & $0.196 \pm 0.041^{\ddagger}$ & $10.5 \pm 0.9$ & $31 \pm 4$ & $0.170 \pm 0.023^{\ddagger}$ & $2.10 \pm 0.39$ \\
\hline
\end{tabular}

FSRs were determined using the monoexponential functions with the VLDL apoB-100 plateau tracer/tracee ratio as the estimate of the precursor pool tracer/tracee ratio for apoA-I and apoA-II. Values for kinetic parameters are given as the best estimate \pm SD. FSR, fractional synthetic rate; PR, production rate. ${ }^{*}$ Value obtained by ${ }^{125}$ I-apoA-I radiotracer study. ${ }^{\ddagger} P<0.01$ compared with the mean of the CETP-deficient homozygotes.

homozygotes, demonstrating that apoA-II has a slower turnover than apoA-I in CETP-deficient as well as in normal subjects. The production rates of apoA-I were within the normal range in both homozygous CETP-deficient subjects. The mean apoA-II production rate in the CETP-deficient homozygotes was $22 \%$ less than that of controls, but was not significantly different $(P=0.27)$. The heterozygous subject had normal apoA-I and apoA-II FSRs and production rates.

The apolipoprotein composition of $\mathrm{HDL}_{1}$ separated by an anti-apoB immunoaffinity column from the $d=1.019-1.063$ $\mathrm{g} / \mathrm{ml}$ lipoproteins of homozygous CETP-deficient subject 2 is illustrated in Fig. 6. The $\mathrm{HDL}_{1}$ was enriched in apoE (apoE/ apoA-I mass ratio $=0.50$ in the CETP-deficient homozygote 1 and 0.82 in the homozygote 2 , compared with 0.02 in controls). ApoA-I was not detectable in LDL (the retained fraction ) by ELISA. The tracer/tracee ratio curves of apoA-I from this apoE-rich $\mathrm{HDL}_{1}$ fraction as well as from $\mathrm{HDL}_{2}$ and $\mathrm{HDL}_{3}$ are shown in Fig. 7, and the kinetic parameters of apoA-I and apoA-II in these HDL subfractions are shown in Table III. Although apoA-I HDL, FSRs were somewhat increased compared with apoA-I HDL 2 FSRs, the difference was not statisti-

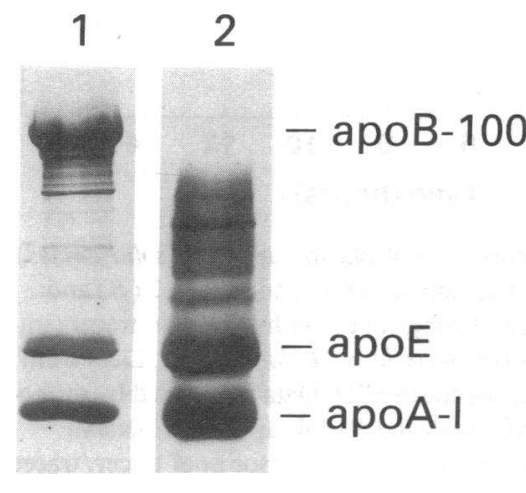

Figure 6. SDS-PAGE of apolipoproteins in lipoproteins from homozygous CETP-deficient subject 2. Lipoproteins $(d=1.019-1.063 \mathrm{~g} / \mathrm{ml}$, lane 1 ) were applied to an anti-apoB-100 immunoaffinity column and the non-retained fraction $\left(\mathrm{HDL}_{1}\right.$, lane 2) was isolated. cally significant $(P=0.10)$. Both the $\mathrm{HDL}_{2}$ and $\mathrm{HDL}_{3}$ apoA-I and apoA-II FSRs were significantly slower in both CETP-deficient homozygotes compared with corresponding FSRs in control subjects, whereas those in the CETP-deficient heterozygote were not different from control subjects.

\section{Discussion}

Although plasma HDL-C levels are highly correlated with plasma apoA-I levels (37), the catabolic rate of HDL-C in humans is much faster than that of apoA-I (38), indicating a dissociation of HDL-C and HDL apolipoprotein metabolism. CETP deficiency in humans results in markedly elevated plasma HDL-C levels, presumably due to the inability to transfer CE from HDL into apoB-containing lipoproteins. However, plasma concentrations of apoA-I are increased in CETP deficiency as well $(14,22)$. The major goal of the present study was to determine the effect of the lack of CETP on the in vivo metabolism of $\mathrm{HDL}$ apolipoproteins A-I and A-II.

We used both endogenous stable isotope and exogenous radiotracer labeling techniques to approach the problem. The use of a primed constant infusion of a stable isotopically labeled amino acid to endogenously label apolipoproteins has been reported by several investigators $(29,39-47)$. This method requires the ability to reliably estimate the tracer/tracee ratio of the precursor pool for apolipoprotein synthesis. The plateau tracer/tracee ratio of VLDL apoB-100, a primarily liver-derived protein, has been used as an estimate for the apoA-I precursor tracer/tracee ratio $(40,41)$. However, because apoA-I is known to be synthesized in the small intestine as well as the liver $(48,49)$, and since evidence suggests that the precursor pool tracer/tracee ratio may not be the same in these two organs $(45,50)$, theoretical concerns have been raised that the VLDL apoB-100 plateau may not serve as a reliable estimate for apoA-I precursor tracer/tracee ratio. We have investi- 


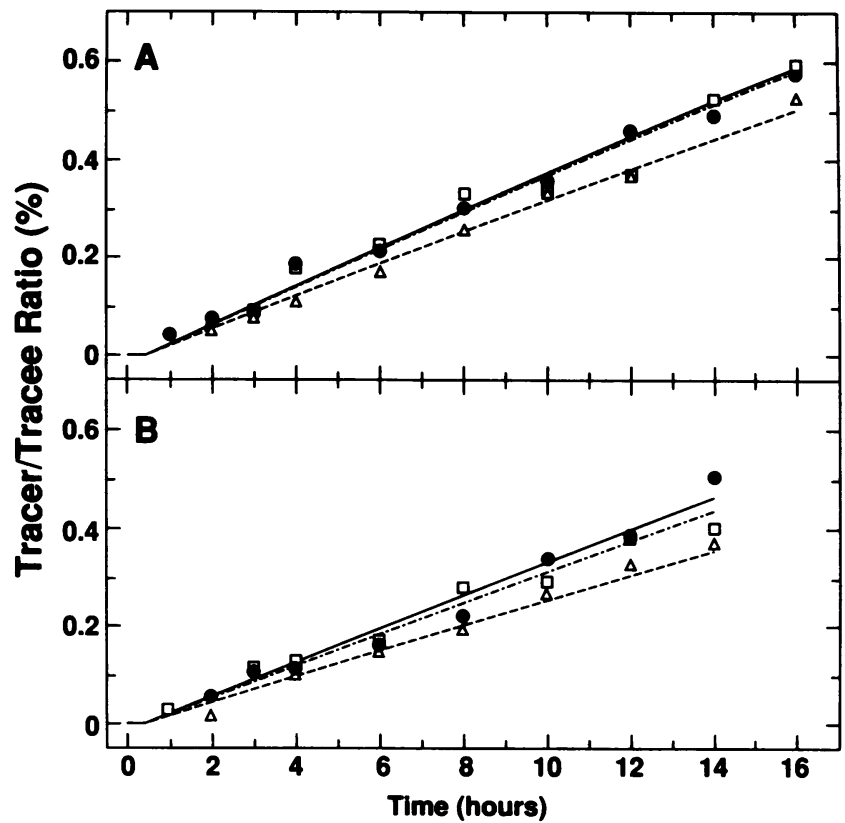

Figure 7. Tracer/tracee ratios of apoA-I in $\mathrm{HDL}_{1}$ (solid circles with solid line), $\mathrm{HDL}_{2}$ (open triangles with dotted line), and $\mathrm{HDL}_{3}$ (open squares with dashed line) in homozygous CETP-deficient subject 1 $(A)$ and subject $2(B)$. Data were fitted by monoexponential function analysis with the VLDL apoB-100 plateau tracer/tracee ratio as the estimate of the precursor pool tracer/tracee ratio for apoA-I.

gated apoA-I kinetics by simultaneous endogenous and exogenous labeling in several subjects with a wide range of plasma apoA-I levels and have found that the use of the VLDL apoB100 plateau tracer/tracee ratio resulted in apoA-I kinetic parameters by endogenous labeling that were highly comparable (only $4 \%$ difference) with those obtained by exogenous radiotracer techniques (Ikewaki, unpublished data). Similarly, in the current study, the apoA-I turnover rates determined by simultaneous endogenous and exogenous labeling in homozygous CETP deficient subject 1 were virtually identical. Moreover, in a primed constant infusion stable isotope study in a patient with extremely low apoA-I, the apoA-I reached a plateau tracer/tracee ratio during the infusion that was $91 \%$ of the VLDL apoB-100 plateau tracer/tracee ratio in the same subject ( $50 \mathrm{a})$. These combined data suggest that the use of the VLDL apoB-100 plateau tracer/tracee ratio as an estimate of the precursor pool tracer/tracee ratio for apoA-I synthesis is a reasonable approach for the determination of apoA-I turnover using primed constant infusion endogenous labeling.

Both CETP-deficient homozygotes were found to have significantly decreased rates of catabolism of apoA-I with normal production rates, indicating that the elevated apoA-I levels were due solely to the delayed apoA-I catabolism. The plasma apoA-II levels were not elevated to the same degree as those of apoA-I, consistent with findings by other investigators $(14,16$, 22 ). However, the CETP-deficient homozygotes also had substantially delayed apoA-II catabolism; the apoA-II production rates were somewhat lower than the controls, but the difference was not statistically significant. Thus, both apoA-I and apoA-II catabolism is delayed in CETP deficiency. It is possible that a secondary decrease in the production rate of apoA-II might partially offset the effect of delayed catabolism, resulting in less marked elevations in plasma apoA-II concentrations.

Our control subjects were somewhat younger than the CETP-deficient subjects. However, in a study by Fidge et al. (51), there was no significant correlation between age and apoA-I FCR ( $r=0.043, P=0.86)$, whereas apoA-I and HDL$C$ levels were inversely correlated with apoA-I FCR's. Given that fact, together with data reported in the Lipid Research Clinic prevalence study (52) and by Stevenson et al. (53) in which HDL-C levels were not different in different age groups, it is highly unlikely that the use of younger controls affected our conclusions. In addition, Hazzard et al. (54) reported that the catabolism of apoA-I in women was delayed by the treatment

Table III. FSRs of apoA-I and apoA-II of $H D L_{1}, H D L_{2}$, and $H D L_{3}$ in Subjects with CETP Deficiency

\begin{tabular}{|c|c|c|c|c|c|}
\hline \multirow[b]{2}{*}{ Subjects } & \multicolumn{3}{|c|}{ ApoA-I } & \multicolumn{2}{|c|}{ ApoA-II } \\
\hline & $\mathrm{HDL}_{1}$ & $\mathrm{HDL}_{2}$ & $\mathrm{HDL}_{3}$ & $\mathrm{HDL}_{2}$ & $\mathrm{HDL}_{3}$ \\
\hline & \multicolumn{5}{|c|}{$d^{-1}$} \\
\hline \multicolumn{6}{|l|}{ CETP deficiency } \\
\hline \multicolumn{6}{|l|}{ Homozygotes } \\
\hline 1 & $0.161 \pm 0.007$ & $0.137 \pm 0.006$ & $0.160 \pm 0.006$ & $0.100 \pm 0.009$ & $0.104 \pm 0.004$ \\
\hline 2 & $0.158 \pm 0.009$ & $0.114 \pm 0.006$ & $0.137 \pm 0.007$ & $0.096 \pm 0.005$ & $0.121 \pm 0.007$ \\
\hline Heterozygote & & $0.228 \pm 0.009$ & $0.240 \pm 0.011$ & $0.139 \pm 0.007$ & $0.185 \pm 0.009$ \\
\hline \multicolumn{6}{|l|}{ Controls } \\
\hline 1 & & $0.242 \pm 0.018$ & $0.270 \pm 0.013$ & $0.176 \pm 0.011$ & $0.202 \pm 0.011$ \\
\hline 2 & & $0.207 \pm 0.007$ & $0.208 \pm 0.006$ & $0.145 \pm 0.007$ & $0.147 \pm 0.006$ \\
\hline 3 & & $0.199 \pm 0.005$ & $0.216 \pm 0.005$ & $0.150 \pm 0.004$ & $0.160 \pm 0.004$ \\
\hline 4 & & $0.213 \pm 0.008$ & $0.220 \pm 0.008$ & $0.155 \pm 0.007$ & $0.166 \pm 0.007$ \\
\hline Mean \pm SD & & $0.215 \pm 0.009^{\ddagger}$ & $0.229 \pm 0.008^{*}$ & $0.156 \pm 0.007^{\ddagger}$ & $0.169 \pm 0.007^{*}$ \\
\hline
\end{tabular}

FSRs were determined using the monoexponential functions with the VLDL apoB-100 plateau tracer/tracee ratio as the estimate of the precursor pool tracer/tracee ratio for apoA-I and apoA-II. Values for FSRs are given as the best estimate \pm SD. ${ }^{*} P<0.05,{ }^{\ddagger} P<0.01$ compared with the mean of the CETP deficient homozygotes. 
with estrogen, which suggests that it is unlikely that the estrogen-deficient postmenopausal status of one of our homozygous subjects (CETP-deficient subject 2) accounted for her delayed apoA-I catabolism.

Our current observations establish that CETP deficiency causes elevated plasma apoA-I and apoA-II levels primarily by modulating their catabolism. However, Brinton et al. (9) reported that female subjects with comparably high plasma apoA-I levels but presumably normal CETP activities had also delayed apoA-I catabolism. Therefore, factors other than CETP deficiency can result in delayed apoA-I catabolism. In addition, we have reported a case with a similarly elevated apoA-I level due to increased apoA-I production (55). Thus, it remains to be determined whether variation in CETP activity plays an important role in regulating plasma apoA-I levels in normal or dyslipidemic populations.

The mechanism of the delayed apoA-I and apoA-II catabolism in humans with CETP deficiency may be related to increased HDL core lipid and particle size. Data from our laboratory $(25)$ and others $(9,56,57)$ suggest that HDL particle size may affect metabolism, with smaller particles having a faster catabolism. Since HDL particles in CETP-deficient homozygotes are extremely enlarged and lipid enriched, the conformation of apoA-I and apoA-II may be altered, resulting in the decreased cellular uptake and catabolism (58). CETP is believed to participate in the conversion of larger $\mathrm{HDL}_{2}$ to smaller $\mathrm{HDL}_{3}(5,59,60)$. During this process, some $\mathrm{HDL}$ apolipoproteins may be transferred to triglyceride-rich lipoproteins (11). Both the conversion to smaller HDL particles and transfer of apolipoproteins to triglyceride-rich lipoproteins may result in faster apolipoprotein catabolism. Since this process is interrupted in CETP deficiency, the relative proportion of apoA-I and apoA-II catabolized by these relatively rapid pathways may be decreased, thus delaying the overall catabolic rates of these apolipoproteins.

Despite the fact that $\mathrm{HDL}_{2}$ is selectively increased in CETP deficiency, the turnover of apoA-I and apoA-II was found to be decreased in both $\mathrm{HDL}_{2}$ and $\mathrm{HDL}_{3}$ in the CETP-deficient homozygotes, indicating that lack of CETP affects HDL apolipoprotein metabolism throughout the entire HDL density spectrum. Furthermore, although the difference was not statistically significant, the catabolism of apoA-I in $\mathrm{HDL}_{1}$ was somewhat faster than that in $\mathrm{HDL}_{2}$ in both CETP-deficient homozygotes, possibly due to the enrichment of apoE on the $\mathrm{HDL}_{1}$ particles. This in vivo evidence is consistent with the in vitro observation that $\mathrm{HDL}_{1}$ from a CETP-deficient subject had greater affinity for the fibroblast LDL receptor than HDL from a normal subject (27). Furthermore, a metabolic study in cholesterol-fed baboons demonstrated that $\mathrm{HDL}_{1}$ was catabolized more rapidly than normal HDL (61). Although speculative, these results suggest that apoE-mediated catabolism of HDL may be one of the pathways for HDL apolipoprotein catabolism (and possibly reverse cholesterol transport) in CETP deficiency. However, the catabolic rates of $\mathrm{HDL}_{1}$ apoA-I in the CETP-deficient homozygotes are still substantially slower than that of normal HDL apoA-I in control subjects. Furthermore, apoE-rich HDL accumulate in the plasma of CETP-deficient subjects, indicating that the effect of the apoE is not enough to fully compensate for the delayed apoA-I catabolism caused by the CETP deficiency.

Although cholesterol distribution in HDL has been shown to be altered in heterozygous CETP deficiency, plasma apoA-I and apoA-II levels vary from normal to slightly higher than normal $(20,22)$. In this study, the heterozygous CETP-deficient subject had normal apoA-I and apoA-II levels and no significant differences in apoA-I and apoA-II metabolism compared with the controls. However, further studies will be required to determine whether heterozygosity for CETP deficiency has an effect on HDL apolipoprotein metabolism or only on HDL-C metabolism.

It has been suggested, but remains to be proven, that the high levels of HDL-C and apoA-I in CETP deficiency may be protective against the development of atherosclerosis $(14,20)$. Recent studies in transgenic mice overexpressing human apoA-I (62) suggest a protective role of increased apoA-I biosynthesis. The present study demonstrates that the elevated levels of apoA-I in CETP deficiency are due solely to delayed catabolism, which may not have the same physiologic effect on reverse cholesterol transport or atherogenesis as increased apoA-I biosynthesis. The elevated HDL-C and apoA-I could exert a protective effect by a mechanism distinct from reverse cholesterol transport, perhaps by preventing the formation of oxidized LDL $(63,64)$. The low plasma LDL cholesterol and apoB levels in CETP-deficient subjects (20) may also play a protective role. Therefore, both increased HDL-C and decreased LDL-C levels could potentially contribute to the decreased risk of atherosclerosis possibly associated with CETP deficiency.

In summary, complete deficiency of CETP results in substantially delayed catabolism of apoA-I and apoA-II with normal production rates of these apolipoproteins. ApoA-I and apoA-II kinetics were normal in a heterozygous CETP-deficient subject. This study provides new insights into the role of CETP in modulating HDL apolipoprotein metabolism and possibly the risk of premature cardiovascular disease.

\section{Acknowledgments}

We are indebted to Dr. Silvia Santamarina-Fojo for support in the PCR amplification and sequencing of the CETP gene; to Marie Kindt, Kathy Parrott, Glenda Tally, and Yoshiko Doherty for excellent technical support; to Bob Butky for quality control of the labeled amino acid; to George Grimes and the Pharmaceutical Development Service for evaluation of injection materials; and to the nursing staffs of the Aoto Hospital, Jikei University School of Medicine, and the National Institutes of Health Clinical Center for nursing care of the study subjects.

\section{References}

1. Miller, G. J., and N. E. Miller. 1975. Plasma-high-density-lipoprotein concentration and development of ischaemic heart-disease. Lancet. i:16-19.

2. Gordon, D. J., and B. M. Rifkind. 1989. High-density lipoprotein-the clinical implications of recent studies. N. Engl. J. Med. 321:1311-1316.

3. Glomset, J. A. 1968. The plasma lecithins:cholesterol acyltransferase reaction. J. Lipid Res. 9:155-167.

4. Reichl, D., and N. E. Miller. 1989. Pathophysiology of reverse cholesterol transport. Insights from inherited disorders of lipoprotein metabolism. Arteriosclerosis. 9:785-797.

5. Tall, A. R. 1990. Plasma high density lipoproteins. Metabolism and relationship to atherogenesis. J. Clin. Invest. 86:379-384.

6. Brewer, H. B., Jr., R. E. Gregg, J. M. Hoeg, and S. S. Fojo. 1988. Apolipoproteins and lipoproteins in human plasma: an overview. Clin. Chem. 34:4-8.

7. Miller, N. E. 1987. Associations of high-density lipoprotein subclasses and apolipoproteins with ischemic heart disease and coronary atherosclerosis. $\mathrm{Am}$. Heart J. 113:589-597.

8. Schaefer, E. J., L. A. Zech, L. L. Jenkins, T. J. Bronzert, E. A. Rubalcaba, 
R. T. Lindgren, R. L. Aamodt, and H. B. Brewer, Jr. 1982. Human apolipoprotein A-I and A-II metabolism. J. Lipid Res. 23:850-862.

9. Brinton, E. A., S. Eisenberg, and J. L. Breslow. 1989. Elevated high density lipoprotein cholesterol levels correlate with decreased apolipoprotein A-I and A-II fractional catabolic rate in women. J. Clin. Invest. 84:262-269.

10. Brinton, E. A., S. Eisenberg, and J. L. Breslow. 1990. A low-fat diet decreases high density lipoprotein (HDL) cholesterol levels by decreasing HDL apolipoprotein transport rates. J. Clin. Invest. 85:144-151.

11. Tall, A. R. 1986. Plasma lipid transfer proteins. J. Lipid Res. 27:361-367.

12. Hesler, C. B., T. L. Swenson, and A. R. Tall. 1987. Purification and characterization of a human plasma cholesteryl ester transfer protein. J. Biol. Chem. 262:2275-2282.

13. Malloy, L. K., J. M. Vandenbroek, L. A. Zech, and C. C. Schwartz. 1990. HDL esterified cholesterol uptake by tissues is negligible in vivo in man. Circulation. 82:III-446. ( Abstr.)

14. Saito, F. 1984. A pedigree of homozygous familial hyperalphalipoproteinemia. Metab. Clin. Exp. 33:629-633.

15. Koizumi, J., H. Mabuchi, A. Yoshimura, I. Michishita, M. Takeda, H. Itoh, Y. Sakai, T. Sakai, K. Ueda, and R. Takeda. 1985. Deficiency of serum cholesteryl-ester transfer activity in patients with familial hyperalphalipoproteinaemia. Atherosclerosis. 58:175-186.

16. Kurasawa, T., S. Yokoyama, Y. Miyake, T. Yamamura, and A. Yamamoto. 1985. Rate of cholesteryl ester transfer between high and low density lipoproteins in human serum and a case with decreased transfer rate in association with hyperalphalipoproteinemia. J. Biochem. (Tokyo). 98:1499-1508.

17. Brown, M. L., A. Inazu, C. B. Hesler, L. B. Agellon, C. Mann, M. E. Whitlock, Y. L. Marcel, R. W. Milne, J. Koizumi, H. Mabuchi, et al. 1989. Molecular basis of lipid transfer protein deficiency in a family with increased high-density lipoproteins. Nature (Lond.). 342:448-451.

18. Tollefson, J. H., and J. J. Albers. 1986. Isolation, characterization, and assay of plasma lipid transfer proteins. Methods Enzymol. 129:797-816.

19. Saiki, R. K., D. H. Gelfand, S. Stoffel, S. J. Scharf, R. Higuchi, G. T. Horn, K. B. Mullis, and H. A. Erlich. 1988. Primer-directed enzymatic amplification of DNA with a thermostable DNA polymerase. Science (Wash. DC). 239:487-491.

20. Inazu, A., M. L. Brown, C. B. Hesler, L. B. Agellon, J. Koizumi, K. Takata, Y. Maruhama, H. Mabuchi, and A. R. Tall. 1990. Increased high-density lipoprotein levels caused by a common cholesteryl-ester transfer protein gene mutation. N. Engl. J. Med. 323:1234-1238.

21. Yamashita, S., D. Y. Hui, D. L. Sprecher, Y. Matsuzawa, N. Sakai. S. Tarui, D. Kaplan, J. R. Wetterau, and J. A. Harmony. 1990. Total deficiency of plasma cholesteryl ester transfer protein in subjects homozygous and heterozygous for the intron 14 splicing defect. Biochem. Biophys. Res. Commun. 170:1346-1351.

22. Yamashita, S., D. Y. Hui, J. R. Wetterau, D. L. Sprecher, J. A. K. Harmony, N. Sakai, Y. Matsuzawa, and S. Tarui. 1991. Characterization of plasma lipoproteins in patients heterozygous for human plasma cholesteryl ester transfer protein (CETP) deficiency: plasma CETP regulates high-density lipoprotein concentration and composition. Metab. Clin. Exp. 40:756-763.

23. Mann, W. A., R. E. Gregg, D. L. Sprecher, and H. B. Brewer, Jr. 1989. Apolipoprotein $\mathrm{E}-\mathrm{I}_{\text {Harisburg: }}$ a new variant of apolipoprotein $\mathrm{E}$ dominantly associated with type III hyperlipoproteinemia. Biochim. Biophys. Acta. 1005:239244.

24. Brewer, H. B., Jr., T. Fairwell, A. LaRue, R. Ronan, A. Houser, and T. J. Bronzert. 1978. The amino acid sequence of human ApoA-I, an apolipoprotein isolated from high density lipoproteins. Biochem. Biophys. Res. Commun. 80:623-630.

25. Rader, D. J., G. Castro, L. A. Zech, J. C. Fruchart, and H. B. Brewer, Jr. 1991. In vivo metabolism of apolipoprotein A-I on high density lipoprotein particles LpA-I and LpA-I, A-II. J. Lipid Res. 32:1849-1859.

26. Havel, R. J., H. A. Eder, and J. H. Bragdon. 1955. The distribution and chemical composition of ultracentrifugally separated lipoproteins in human serum. J. Clin. Invest. 34:1345-1353.

27. Yamashita, S., D. L. Sprecher, N. Sakai, Y. Matsuzawa, S. Tarui, and D. Y. Hui. 1990. Accumulation of apolipoprotein E-rich high density lipoproteins in hyperalphalipoproteinemic human subjects with plasma cholesteryl ester transfer protein deficiency. J. Clin. Invest. 86:688-695.

28. Bisgaier, C. L., M. V. Siebenkas, M. L. Brown, A. Inazu, J. Koizumi, H. Mabuchi, and A. R. Tall. 1991. Familial cholesteryl ester transfer protein deficiency is associated with triglyceride-rich low density lipoproteins containing cholesteryl esters of probable intracellular origin. J. Lipid Res. 32:21-33.

29. Schaefer, J. R., D. J. Rader, R. E. Gregg, T. Fairwell, L. A. Zech, M. R. Kindt, M. D. Benson, and H. B. Brewer, Jr. 1990. In vivo protein metabolism utilizing stable isotopes and mass spectrometry: a new approach to the study of mutant proteins in humans. Trans. Assoc. Am. Physicians. CIII:187-194.

30. Brewer, H. B., Jr., T. Fairwell, M. Meng, L. Kay, and R. Ronan. 1983. Human proapoA-ITangier: isolation of proapoA-I $\mathrm{I}_{\text {Tangier }}$ and amino acid sequence of the propeptide. Biochem. Biophys. Res. Commun. 113:934-940.

31. Cobelli, C., G. Toffolo, and D. M. Foster. 1992. Tracer-to-tracee ratio for analysis of stable isotope tracer data: link with radioactive kinetic formalism. Am. J. Physiol. 262:E968-E975.

32. Berman, M., and M. Weiss. 1978. SAAM Manual. DHEW Publ. No. (NIH) 78:180. National Institutes of Health. Bethesda, MD

33. Kesaniemi, Y. A., G. L. Vega, and S. M. Grundy. 1982. Kinetics of apolipoprotein B in normal and hyperlipidemic man: review of current data. In Lipoprotein Kinetics and Modeling. M. Berman, S. M. Grundy, and B. V. Howards, editors. Academic Press, New York. 181-205.

34. Wolfe, R. R. 1984. Whole body protein turnover. In Tracers in Metabolic Research. Radioisotope and Stable Isotope/Mass Spectrometry Methods. R. R. Wolfe, editor. Alan R. Liss, Inc., New York. 157-173.

35. Warnick, G. R., M. C. Cheung, and J. J. Albers. 1979. Comparison of current methods for high-density lipoprotein cholesterol quantitation. Clin. Chem. 25:596-604.

36. Bojanovski, M., R. E. Gregg, D. M. Wilson, and H. B. Brewer, Jr. 1988. Competitive enzyme-linked immunosorbent assay (ELISA) for the quantitation of apolipoprotein A-I using a monoclonal antibody. Clin. Chim. Acta. 178:159169.

37. Schaefer, E. J., W. H. Heaton, M. G. Wetzel, and H. B. Brewer, Jr. 1982. Plasma apolipoprotein A-1 absence associated with a marked reduction of high density lipoproteins and premature coronary artery disease. Arteriosclerosis. 2:16-26.

38. Schwartz, C. C., M. Berman, Z. R. Vlahcevic, and L. Swell. 1982. Multicompartmental analysis of cholesterol metabolism in man. Quantitative kinetic evaluation of precursor sources and turnover of high density lipoprotein cholesterol esters. J. Clin. Invest. 70:863-876.

39. Cryer, D. R., T. Matsushima, J. B. Marsh, M. Yudkoff, P. M. Coates, and J. A. Cortner. 1986. Direct measurement of apolipoprotein B synthesis in human very low density lipoprotein using stable isotopes and mass spectrometry. J. Lipid Res. 27:508-516.

40. Cohn, J. S., D. A. Wagner, S. D. Cohn, J. S. Millar, and E. J. Schaefer. 1990. Measurement of very low density and low density lipoprotein apolipoprotein (Apo) B-100 and high density lipoprotein Apo A-I production in human subjects using deuterated leucine. Effect of fasting and feeding. J. Clin. Invest. $85: 804-811$

41. Lichtenstein, A. H.. J. S. Cohn, D. L. Hachey, J. S. Millar, J. M. Ordovas, and E. J. Schaefer. 1990. Comparison of deuterated leucine, valine, and lysine in the measurement of human apolipoprotein A-I and B-100 kinetics. J. Lipid Res. 31:1693-1701.

42. Parhofer, K. G., P. H. R. Barrett, D. M. Bier, and G. Schonfeld. 1991. Determination of kinetic parameters of apolipoprotein B metabolism using amino acids labeled with stable isotopes. J. Lipid Res. 32:1311-1323.

43. Walsh, B. W., I. Schiff, B. Rosner, L. Greenberg, V. Ravnikar, and F. M. Sacks. 1991. Effects of postmenopausal estrogen replacement on the concentrations and metabolism of plasma lipoproteins. N. Engl. J. Med. 325:1196-1204.

44. Parhofer, K. G., P. H. R. Barrett, D. M. Bier, and G. Schonfeld. 1992. Lipoproteins containing the truncated apolipoprotein, apo B-89, are cleared from human plasma more rapidly than apo B-100-containing lipoproteins in vivo. $J$. Clin. Invest. 89:1931-1937.

45. Lichtenstein, A. H., D. L. Hachey, J. S. Millar, J. L. Jenner, L. Booth, J. Ordovas, and E. J. Schaefer. 1992. Measurement of human apolipoprotein B-48 and B-100 kinetics in triglyceride-rich lipoproteins using $\left[5,5,5-{ }^{2} \mathrm{H}_{3}\right]$ leucine. $J$. Lipid Res. 33:907-914.

46. Krul, E. S., K. G. Parhofer, P. H. R. Barrett, R. D. Wagner, and G. Schonfeld. 1992. ApoB-75, a truncation of apolipoprotein B associated with familial hypobetalipoproteinemia: genetic and kinetic studies. J. Lipid Res 33:1037-1050.

47. Schaefer, J. R., D. J. Rader, and H. B. Brewer, Jr. 1992. Investigation of lipoprotein kinetics using endogenous labeling with stable isotopes. Curr. Opin. Lipidol. 3:227-232.

48. Hopf, U., G. Assmann, H. E. Schaefer, and A. Capurso. 1979. Demonstration of human apolipoprotein $\mathrm{A}$ in isolated mucosal cells from small intestine and isolated hepatocytes. Gut. 20:219-25.

49. Bisgaier, C. L., and R. M. Glickman. 1983. Intestinal synthesis, secretion, and transport of lipoproteins. Annu. Rev. Physiol. 45:625-636.

50. Schaefer, J. R., D. J. Rader, R. E. Gregg, F. Thomas, M. R. Kindt, L. A. Zech, and H. B. Brewer, Jr. 1990. In vivo apoB-100 and apoA-I kinetics in Tangier disease utilizing a stable isotope technique. Circulation. 82:III-447. (Abstr.)

50a.Emmerich J. E., B. Verges, I. Tauveron, D. J. Rader, S. SantamarinaFojo, J. Schaefer, M. Ayrault-Jarrier, P. Thieblot, and H. B. Brewer, Jr. 1993. Familial high density lipoprotein deficiency due to marked hypercatabolism of normal apolipoprotein A-1. Arterioscler. Thromb. In press.

51. Fidge, N., P. Nestel, T. Ishikawa, M. Reardon, and T. Billington. 1980 Turnover of apoproteins A-I and A-II of high density lipoprotein and the relationship to other lipoproteins in normal and hyperlipidemic individuals. Metab. Clin. Exp. 29:643-653.

52. Heiss, G., I. Tamir, C. E. Davis, H. A. Tyroler, B. M. Rifkand, G. Schon- 
feld, D. Jacobs, and I. D. Frantz, Jr. 1980. Lipoprotein-cholesterol distributions in selected North American populations: the lipid research clinics program prevalence study. Circulation. 61:302-15.

53. Stevenson, J. C., D. Crook, and I. F. Godsland. 1993. Influence of age and menopause on serum lipids and lipoproteins in healthy women. Atherosclerosis. 98:83-90.

54. Hazzard, W. R., S. M. Haffner, R. S. Kushwaha, D. Applebaum-Bowden and D. M. Foster. 1984. Preliminary report: kinetic studies on the modulation of high-density lipoprotein, apolipoprotein, and subfraction metabolism by sex steroids in a postmenopausal woman. Metab. Clin. Exp. 33:779-784.

55. Rader, D. J., J. R. Schaefer, P. Lohse, K. Ikewaki, F. Thomas, W. A. Harris, L. A. Zech, C. A. Dujovne, and H. B. Brewer, Jr. 1993. Increased production of apolipoprotein A-I associated with elevated with plasma levels of high density lipoproteins and apolipoproteins A-I in a patient with familial hyperalphalipoproteinemia. Metab. Clin. Exp. In press.

56. Brinton, E. A., S. Eisenberg, and J. L. Breslow. 1991. Increased apo A-I and apo A-II fractional catabolic rate in patients with low high density lipoprotein-cholesterol levels with or without hypertriglyceridemia. J. Clin. Invest. 87:536-544.

57. Horowitz, B. S., I. J. Goldberg, J. Merab, T. Vanni, R. Ramakrishnan, and H. N. Ginsberg. 1991. Increased plasma and renal clearance of an exchangeable pool of apolipoprotein A-I in subjects with low levels of high density lipoprotein cholesterol. J. Clin. Invest. 91:1743-1752.
58. Collet, X., B. Perret, G. Simard, E. Raffaï, and Y. L. Marcel. 1991. Differential effects of lecithin and cholesterol on the immunoreactivity and conformation of apolipoprotein A-I in high density lipoproteins. J. Biol. Chem. 266:91459152.

59. Barter, P. J., O. V. Rajaram, L. B. Chang, K. A. Rye, P. Gambert, L. Lagrost, C. Ehnholm, and N. H. Fidge. 1988. Isolation of a high-density-lipoprotein conversion factor from human plasma. A possible role of apolipoprotein A-IV as its activator. Biochem. J. 254:179-184.

60. Lagrost, L., and P. J. Barter. 1991. Effects of various non-esterified fatty acids on the particle size redistribution of high density lipoproteins induced by the human cholesteryl ester transfer protein. Biochim. Biophys. Acta. 1082:204-210.

61. Kushwaha, R. S., D. M. Foster, V. N. Murthy, K. D. Carey, and H. C. McGill, Jr. 1989. Metabolism of larger high density lipoproteins accumulating in some families of baboons fed a high cholesterol and high saturated fat diet. $J$. Lipid Res. 30:1147-1159.

62. Rubin, E. M., R. M. Krauss, E. A. Spangler, J. G. Verstuyft, and S. M. Clift. 1991. Inhibition of early atherogenesis in transgenic mice by human apolipoprotein AI. Nature (Lond.). 353:265-267.

63. Witztum, J. L., and D. Steinberg. 1991. Role of oxidized low density lipoprotein in atherogenesis. J. Clin. Invest. 88:1785-1792.

64. Parthasarathy, S., J. Barnett, and L. G. Fong. 1990. High-density lipoprotein inhibits the oxidative modification of low-density lipoprotein. Biochim. Biophys. Acta. 1044:275-283. 